\title{
Les vanneries natives en Bretagne, contribution à la délimitation d'aires culturelles de l'Armorique ancienne
}

Native basketry in Brittany: contribution to the delimitation of cultural areas in ancient Armorica

\section{Roger Hérisset}

\section{OpenEdition}

\section{Journals}

Édition électronique

URL : https://journals.openedition.org//bl/311

DOl : $10.4000 / \mathrm{lbl} .311$

ISSN : 2727-9383

Éditeur

Université de Bretagne Occidentale - UBO

\section{Édition imprimée}

Date de publication : 1 mai 2017

Pagination : 111-136

ISBN : 979-10-92331-31-8

ISSN : $1270-2412$

\section{Référence électronique}

Roger Hérisset, «Les vanneries natives en Bretagne, contribution à la délimitation d'aires culturelles de l'Armorique ancienne », La Bretagne Linguistique [En ligne], 21 | 2017, mis en ligne le 01 mai 2020 consulté le 23 mars 2022. URL : http://journals.openedition.org/lbl/311 ; DOI : https://doi.org/ 10.4000//bl.311

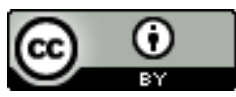

La Bretagne Linguistique est mise à disposition selon les termes de la Licence Creative Commons Attribution 4.0 International. 


\title{
Les vanneries natives en Bretagne, contribution à la délimitation d'aires culturelles de l'Armorique ancienne
}

\begin{abstract}
La vannerie en Bretagne a fait l'objet d'une étude détaillée ${ }^{1}$. Des hypothèses quant à la dynamique de diffusion de différentes techniques y ont été formulées. Il est proposé ici de rapprocher la cartographie actuelle des types de vanneries régionales avec des représentations d'aires techniques de la Protohistoire et de la période gallo-romaine. Ces rapprochements permettent d'esquisser des aires de diffusion culturelle, dont la permanence s'observe dans les techniques de vannerie et dans le vocabulaire désignant les objets produits.

L'analyse s'appuie principalement sur les vanneries à montants courbés. Cette technique n'a pas fait l'objet de description ethnographique, à l'exception d'une étude des vanniers de Mayun en Brière et ceux de Cancale $^{2}$. Les vanneries à montants droits, dont la diffusion est plus récente en Bretagne, ne sont pas considérées ici. L'étude des vanneries à montant spiralé (constituée par un cordon de graminées
\end{abstract}

* Docteur en ethnologie, chercheur associé au CRBC (EA 4451)

1. Roger HÉRISSET, Ethnologie des techniques de tressage en Bretagne. Matériaux pour une nouvelle approche classificatoire, thèse CRBC-UBO, Brest, 2012.

2. Dan LALLIER, "Pierre Lelièvre, vannier à La-Chapelle-des-Marais", Monographies d'entreprises artisanales, «Chantier 1810 », n 8, G. H. Rivière et M. Maget (dir.), 1942 et D. LALLIER, «Les vanniers de Cancale, la vannerie pour la grande pêche », Monographies d'entreprises artisanales, « Chantier 1810 », n 58, G. H. Rivière et M. Maget (dir.), 1952. 
enroulé) apporte un éclairage complémentaire qui assoit les hypothèses formulées.

\section{Modèle descriptif des vanneries}

Le système descriptif de la vannerie proposé s'appuie sur le mode d'utilisation et d'organisation des montants, lesquels sont les brins participant à l'ossature. Ce système permet de distinguer les vanneries à montants courbés disposés en hémiméridien organisés autour d'un pôle unique (fig. 1 et 2), de celles à montants courbés disposés en méridien reliant au moins deux pôles (fig. 3 et 4 ).

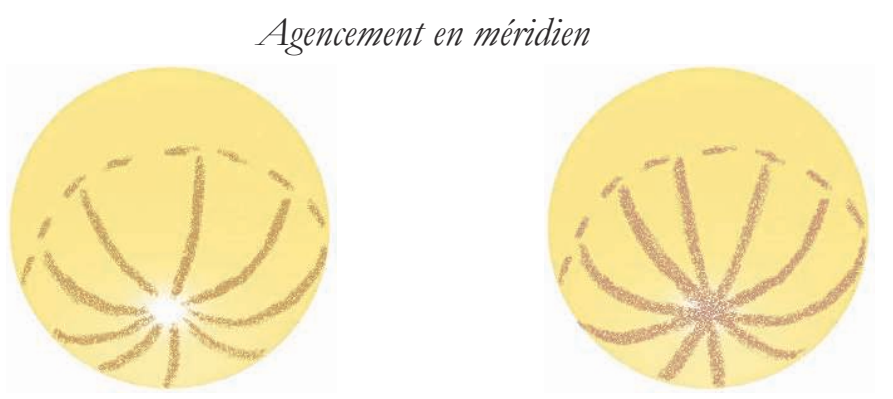

Fig. 1 - Convergence centrale unipolaire

Fig. 2 : Croisement central unipolaire.

Agencement en hémiméridien

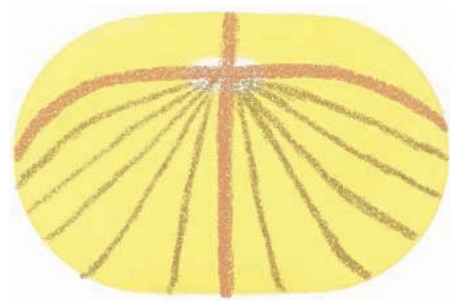

Fig. 3 : Convergence bipolaire combinée à un croisement orthogonal.

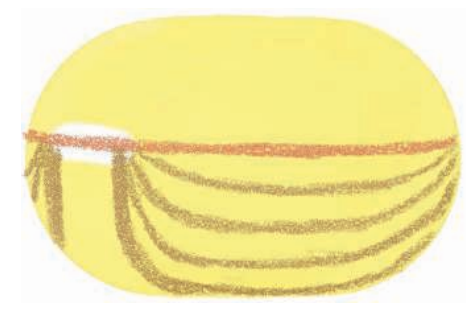

Fig. 4 : Convergence bipolaire à montants convergents et parallèles.

\section{La vannerie à montants courbés disposés en hémiméridien}

La vannerie à montants passifs courbés en hémiméridien est probablement un type natif. Les mots désignant localement la vannerie de ce groupe n'ont pas un sens générique. À l'inverse, un panier fabriqué avec une autre technique est le plus souvent appelé avec le terme géné- 
rique emprunté au français panier, sous la forme penier dans les dialectes romans et paner dans les dialectes celtiques.

La vannerie native peut être séparée en deux groupes techniques distincts. Un premier groupe présente des montants concentriques en arcs rampants. Des exemples sont présentés par les figures 5 à 7 .

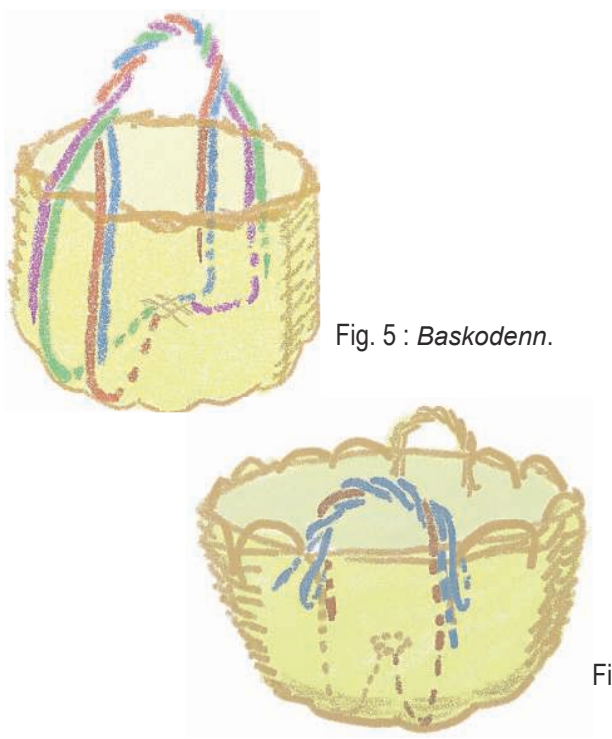

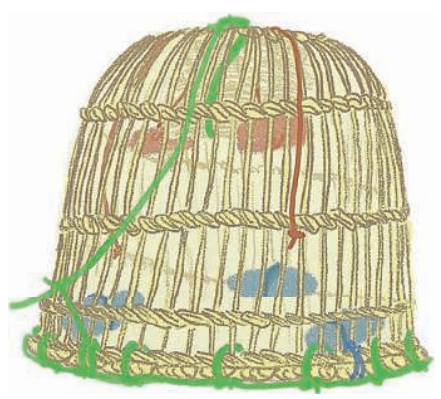

Fig. 7 : Kawell mor.

Un second groupe est constitué de vanneries présentant des montants croisés en lames de bois (ex. fig. 8 à 10). Elles se présentent sous la forme de corbeilles aux montants composés de lames de châtaignier. Leur clôture est confectionnée en bourdaine.

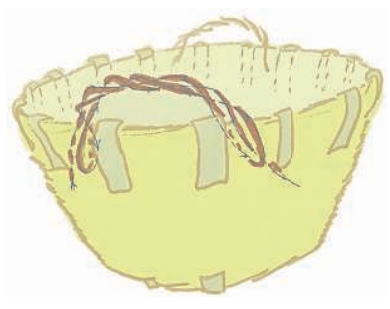

Fig. 8 : Carbasson.

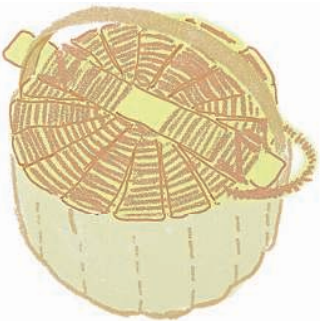

Fig. 9 : Panier à anguilles de Mayun.

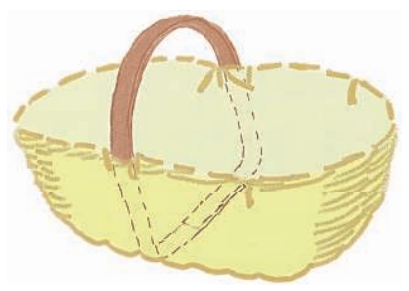

Fig. 10 : Panier de Mayun.

La carte 1 présente les aires de production de la vannerie à montants passifs courbés disposés en hémiméridien. 


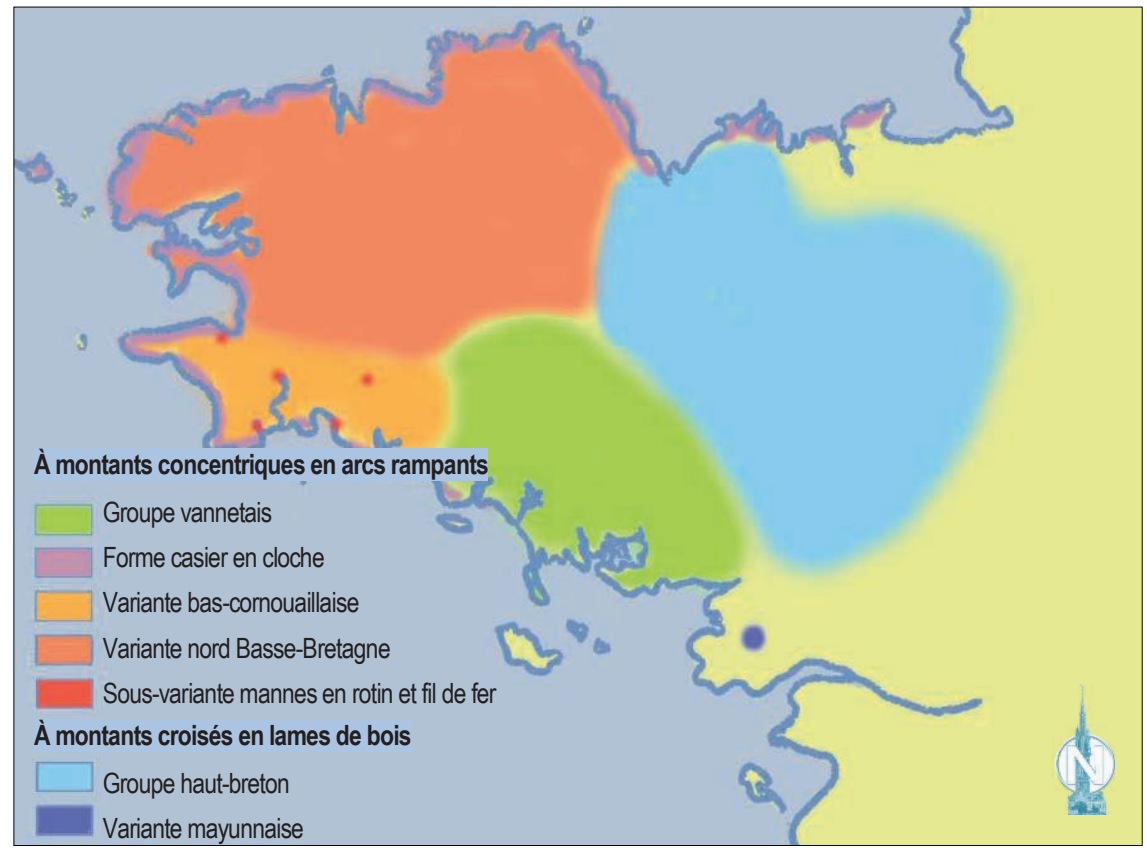

Carte 1 : Aire de production de la vannerie à montants passifs courbés en hémiméridien.

La vannerie à montants croisés en lames de bois est produite au nord-est de la Bretagne dans un secteur de parlers romans. Il s'agit d'une production essentiellement domestique. Dans ce secteur, les corbeilles sont confectionnées sur l'ensemble de la zone de production de manière normalisée : mêmes dimensions, même mode de fabrication (seize montants...) et mêmes matériaux de construction (bourdaine et châtaignier), ce qui est le signe d'une certaine homogénéité culturelle. L'aire de production de ces corbeilles rassemble toutefois plusieurs groupes géolinguistiques, aux territoires bien délimités. Plus au sud, le village briéron de Mayun confectionne une variante avec des brins plus affinés.

À l'ouest, la vannerie à montants concentriques en arcs rampants est pratiquée dans les régions de parlers celtiques. La vannerie du Vannetais constitue un groupe défini par un nombre impair de montants, rayonnant à partir d'un bouton de bois entortillé. Le mode d'arrêt y est très caractéristique : les montants sont renversés et piqués le long d'un montant voisin. 
Le secteur sud-cornouaillais se distingue notamment par un mode d'arrêt plus élaboré, à montants pliés puis enchevêtrés, et un nombre toujours impair de montants. Dans la seconde partie du XXe siècle, une sous-variante " manne en rotin et fil de fer » est apparue dans les ateliers travaillant pour les marins-pêcheurs. Le reste du secteur «nord Basse-Bretagne » partage certains éléments techniques avec la variante «bas-cornouaillaise », mais avec des formes techniques plus variées. Ces variantes forment le groupe de vannerie « ouest-breton».

La carte 2 présente la variété des formes et la localisation des casiers à crustacés en forme de cloche.

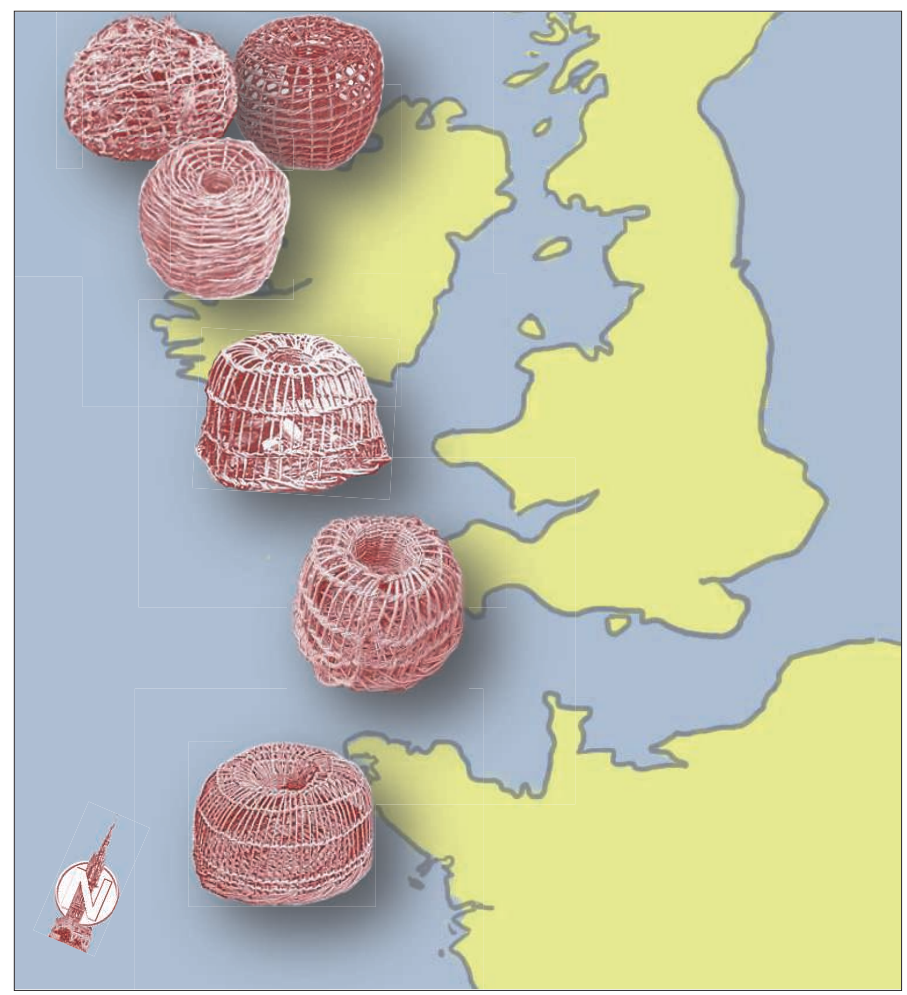

Carte 2 : Localisation des différents types de casiers en cloche.

Les casiers en cloche réalisés dans le sud de l'Irlande s'apparentent à ceux produits en Bretagne et dans le Devon, contrairement à ceux 
fabriqués sur la côte ouest-irlandaise, beaucoup plus grossiers ${ }^{3}$. Ainsi, ce type de nasse, appelé en breton kawell mor, pourrait être l'archétype des groupes de vannerie « vannetais » et « ouest-breton». Cette forme technique a pu diffuser de la Bretagne vers les Cornouailles britanniques et le sud de l'Irlande.

\section{La vannerie à montants courbés disposés en méridien}

La vannerie à montants passifs courbés disposés en méridien est appelée par les vanniers "vannerie sur côtes » ou encore "vannerie bâtie sur arceaux ». Le tressage est réalisé sur une monture préalablement construite à partir de montants courbés en arc, fixés à partir de l'anse ou du bord. La carte 3 montre que ce groupe technique est pratiqué essentiellement à l'est et au sud de la Bretagne. Il se répand vers l'ouest en longeant le littoral.

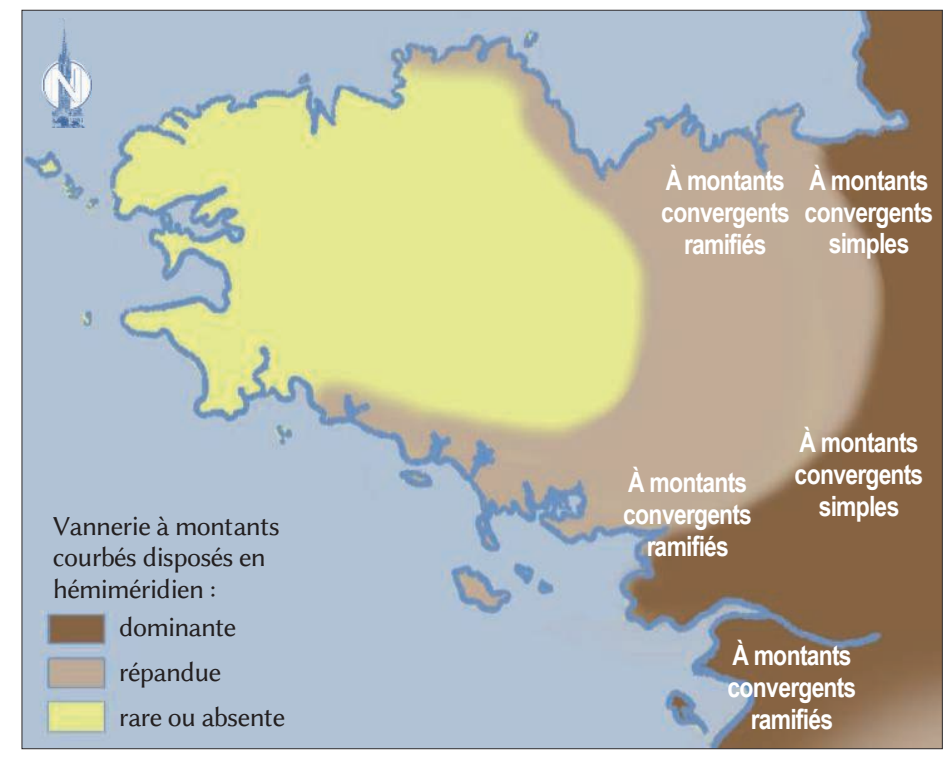

Carte 3 : Les aires bretonnes des vanneries à montants courbés disposés en hémiméridien.

3. Joe HogAn, Basketmaking in Ireland, Wickloow, Wordwell ltd, 2001, p. 114. 
Deux formes d'ossatures produites par des groupes de fabricants distincts ont été observées. La première forme présente des arceaux qui convergent vers deux pôles opposés (fig. 11 à 14).

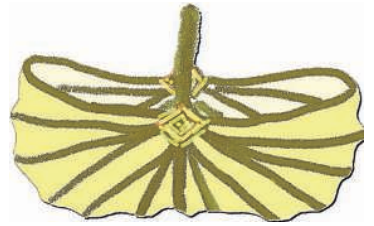

Fig. 11 : Panier à pommes.

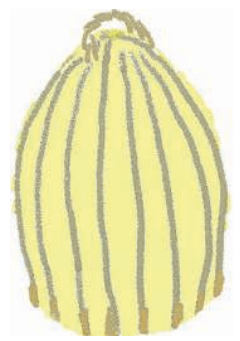

Fig. 13 : Ruche.

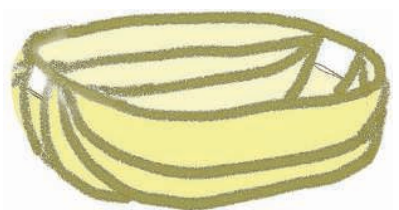

Fig. 12 : Resse.

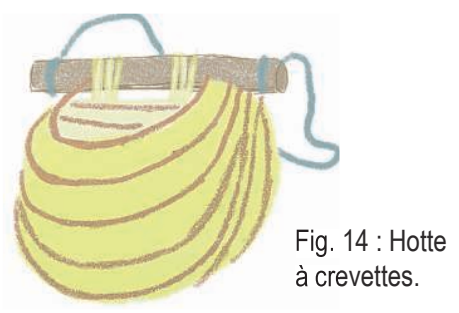

Cette vannerie à monture convergente bipolaire simple existe également dans les régions limitrophes. Elle se localise dans la frange nordest de la Haute-Bretagne où elle domine en répondant à tous les types d'usages, et sous de nombreuses formes : paniers, grandes corbeilles agraires, ruches, hottes... C'est un artisanat réalisé par des fabricants spécialisés, et lié, par exemple, à la pêche côtière et au ramassage des pommes pour la fabrication du cidre.

La seconde forme comporte des arceaux principaux qui convergent vers deux pôles opposés, et des arceaux secondaires fixés le long des arceaux principaux (fig. 15 à 17). La vannerie à monture convergente bipolaire ramifiée est très fréquente au sud-est de la Bretagne. Elle se rencontre aussi du centre-est Bretagne jusqu'à la côte nord. Il s'agit plutôt d'une vannerie domestique.

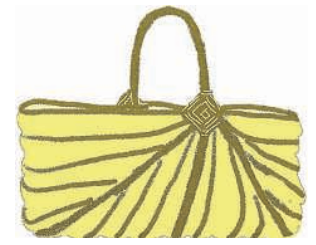

Fig. 15 : Panier.

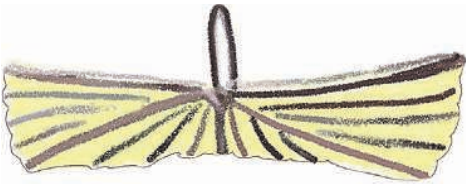

Fig. 16 : Panier à maquereaux.

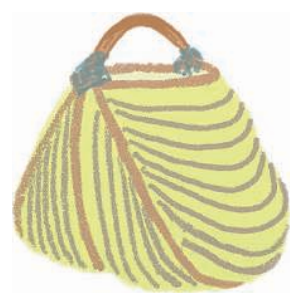

Fig. 17 : Panier à crabes. 


\section{De la permanence de la vannerie native en Bretagne}

L'art de la vannerie a pour particularité d'être peu sensible au progrès technique. De nos jours, les vanniers produisent des objets semblables à ceux fabriqués il y a plusieurs milliers d'années. L'absence en Bretagne d'artefacts archéologiques de vanneries préhistoriques, antiques et même médiévales ne nous permet pas de statuer quant à la permanence géographique de ces techniques. Les milieux favorables à la préservation de la vannerie sont bien connus ${ }^{4}$. Avec des sols acides et des conditions humides, le contexte pédoclimatique armoricain est défavorable à la conservation des objets en bois. A contrario, le climat sec du Proche-Orient a livré de nombreux objets tressés, dont certains produits il y a 10000 ans $^{5}$. La vannerie égyptienne antique est proche des vanneries confectionnées dans les villages voisins des sites archéologiques ${ }^{6}$. À l'instar des observations réalisées au Proche-Orient et en Afrique, la persistance technique des vanneries natives dans l'espace armoricain semble recevable.

La vannerie présente une inertie technique d'exception au regard d'autres artisanats tels que la céramique ou le textile sensibles au progrès technique. En Bretagne, les ateliers se situent préférablement en bordure des massifs forestiers, dans lesquels sont prélevés les matériaux nécessaires à la confection. Ces populations, que l'on peut qualifier de forestières, sont peu mobiles par le fait du lien de leur activité au milieu naturel. Elles forment un groupe culturel à part entière ${ }^{7}$, moins soumis aux évolutions générales en raison d'un mode de vie plus isolée. La vannerie est transmise au sein de lignées familiales, ce qui renforce la stabilité du comportement culturel et technique. Tout ceci contribue au maintien de savoir-faire et de vocabulaires spécifiques.

4. Hélène BALFET, «Vannerie », Dictionnaire archéologique des techniques, Paris, Éditions de l'accueil, 1964.

5. Danielle STORDEuR, «Vannerie et tissage au Proche-Orient néolithique », dans Tissage Corderie Vannerie, APDCA, 1989.

6. Willeke WENDRICH, The World According to Basketry, An Ethno-archaeological interpretation of basketry production in Egypt, CNWS Leiden Universiteit, 1999.

7. Suzanne LE RouzIC, Matériaux ethnographiques et linguistiques pour la connaissance d'une population rurale de Basse-Bretagne : les riverains des forets domaniales de Camors - Floranges Lanvaux (Morbihan) et leur rapport à l'espace boisé, thèse CRBC-UBO, 2007. 
Il est remarquable que les types fréquents sur le continent européen (notamment ceux présentant des ossatures disposées en méridien) soient absents de l'ouest de la Bretagne, alors même qu'ils sont pratiqués en Irlande ou dans les îles britanniques.

La relation probable entre les espaces vanniers contemporains et les aires culturelles anciennes repose donc d'une part sur la continuité des savoirs vanniers dans un espace donné et d'autre part sur leurs singularités dans le contexte européen. Voyons maintenant si des croisements avec des cartographies anciennes permettent d'étayer cette hypothèse.

\section{Les composantes culturelles de l'âge du Bronze...}

En ce qui concerne le Bronze moyen, les chercheurs de l'Inrap Cyril Marcigny et Marc Talon ${ }^{8}$, ont présenté un bilan des recherches archéologiques portant sur les connexions culturelles possibles entre le sud de l'île de Bretagne et le littoral continental de la Normandie jusqu'aux Flandres. La carte 4 reprend une proposition de délimitation sommaire de ces espaces culturels.

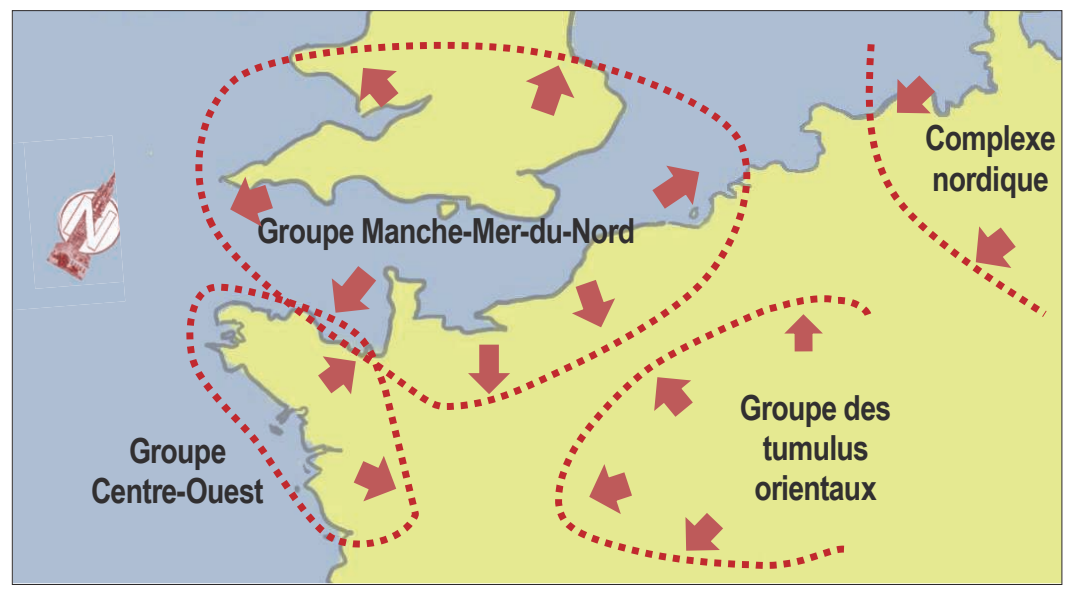

Carte 4 : Localisation des groupes culturels à l'âge du Bronze moyen.

8. Cyril Marcigny, Marc TALON, «Sur les rives de la Manche. Qu'en est-il du passage de l'âge du Bronze à l'âge du Fer à partir des découvertes récentes ? ", De l'âge du Bronze à l'age du Fer en France et en Europe occidentale (Xe-VII av. J.-C.). La moyenne vallée du Rhône aux ages du Fer. Actes du XXXe colloque international de l'AFEAF, 2009. 
L'espace de partage technique transmanche est appelé par les auteurs Composante culturelle Manche-Mer-du-Nord. Cette cartographie s'appuie dans sa définition sur la comparaison des types de céramiques, d'habitat (architecture et organisation) et de domaine funéraire.

S'agissant de la péninsule armoricaine, les recherches archéologiques de l'Inrap portant sur les céramiques provenant de sites du Bronze moyen semblent indiquer qu'il existe peu ou pas d'affinités avec les productions normandes ou britanniques de la Composante culturelle Manche-Mer-du-Nord. La péninsule armoricaine présente plutôt des concordances typologiques voire stylistiques pour un certain nombre de formes et de décors avec le Groupe Centre-Ouest ${ }^{9}$. Toutefois, la Composante culturelle Manche-Mer-du-Nord a une influence probable au nordouest de la péninsule. Un bilan synthétique portant sur l'habitat et l'occupation du sol à l'âge du Bronze en Bretagne est en préparation ${ }^{10}$.

\section{... et concordance avec les groupes techniques de vannerie}

Bien que nous ne puissions pas connaitre avec certitude les techniques de vannerie pratiquées il y a 3000 ans, appuyons-nous sur leur supposée pérennité pour comparer les périmètres culturels du bronze avec les groupes de vanneries régionaux contemporains. À l'instar du Groupe Centre-Ouest, la vannerie à monture disposée en hémiméridien s'inscrit actuellement exclusivement sur le territoire de la péninsule armoricaine, et à son extrémité ouest ce type est l'unique mode de production de vannerie. Notons que cette aire de fabrication s'étendait possiblement dans un espace plus large que la Bretagne historique. Il se trouve un exemple de confection rare, situé au sud de l'aire géographique du Groupe Centre-Ouest. Il s'agit de la production d'une corbeille appelée melon ${ }^{11}$, utilisée pour sécher (meler en poitevin) au four les prunes. Elle comprend seize montants disposés en hémiméridien en lames de châtaignier courbées croisées. Produite aux confins des

\footnotetext{
9. Stéphane Blanchet (dir.), Plouedern (Finistère) - Leslouc'b, Une longue occupation de la Protobistoire au Moyen-âge, Inrap, 2013.

10. S. Blanchet, M. Fily, T. Nicolas, L'habitat et l'occupation du sol à l'âge du Bronze : Premier bilan synthétique pour la Bretagne, s. 1., s. d.

11. Martine Jaoul, Bernadette Goldstein, La Vannerie française, Paris, Musée national des arts et traditions populaires, 1990, p. 193.
} 
départements de la Vendée et des Deux-Sèvres, elle concorderait dans sa forme technique avec la vannerie produite en Haute-Bretagne, et comporterait la même répartition entre le nombre de montants croisés initialement et le nombre de montants rajoutés.

Sur le secteur de la Composante culturelle Manche-Mer-du-Nord, des fabricants produisent de nos jours de la vannerie à monture en méridien à convergence bipolaire simple. Cette vannerie est présente de part et d'autre de la Manche : Normandie, Maine, Marches de Bretagne, Angleterre, Pays de Galles et Irlande.

Au sud et à l'est des deux espaces déjà cités, il est fabriqué une autre variante de la vannerie à montants disposés en méridien. Ceci contribue à la mise en lumière d'un lien culturel plus fort entre l'espace MancheMer-du-Nord et les groupes continentaux qu'avec le Groupe Centre-Ouest, plus singulier culturellement.

\section{La fin de l’âge du fer}

Les habitats de la fin de l'âge du fer situés à l'est de la péninsule armoricaine présentent certains types de céramiques comme les vases tronconiques à lèvre en « $\mathrm{Y}$ ». Ce fait, associé à la présence de "plaques de cuisson », peut être utilisé comme indicateur pour différencier deux cultures matérielles dites « ensemble occidental et ensemble oriental » ${ }^{12}$, présentés sur la carte 5 .

Les sites orientaux de la fin de l'âge du fer se distinguent par la présence de grands conteneurs d'un type fréquent dans les contextes tardifs de la région de Rennes ou bien encore à travers l'emploi de "plaques de cuisson». Les sites les plus occidentaux ayant livré ces plaques sont les sites de la Tourelle, à proximité de Lamballe ${ }^{13}$ et de Bellevue, situé à Augan ${ }^{14}$ représentée sur la carte 5 par les points (1) et (2).

12. Elven LE GOFF, " "Armorique" et "Bretagne” : réflexion sur l'assimilation de ces concepts pour la fin de l'âge du fer ", Revue Archéologique de l'Ouest, supplément n 10, 2003.

13. Stéphane Blanchet (dir.), Lamballe, Côtes-d'Armor, ZAC de la Tourelle, Une occupation de longue durée sur le plateau de la Tourelle, Paris, Inrap, 2010.

14. S. Hinguant, E. Le GofF, S. Jean et D. Marguerie, « Le site gaulois de Bellevue à Augan (Morbihan). Un établissement rural en limite de deux influences armoricaines », Revue Archéologique de l'Ouest, n 14, 1997, p. 57-80. 
Cette partition supposée montrerait une division de la péninsule armoricaine de l'âge du fer en deux espaces d'échanges culturels. Aujourd'hui, les types natifs de vannerie en hémiméridien (carte 1) se partagent des espaces correspondants. Les types à montants concentriques en arcs rampants sont situés à l'ouest et les types à montants croisés en lames de bois à l'est de la Bretagne.

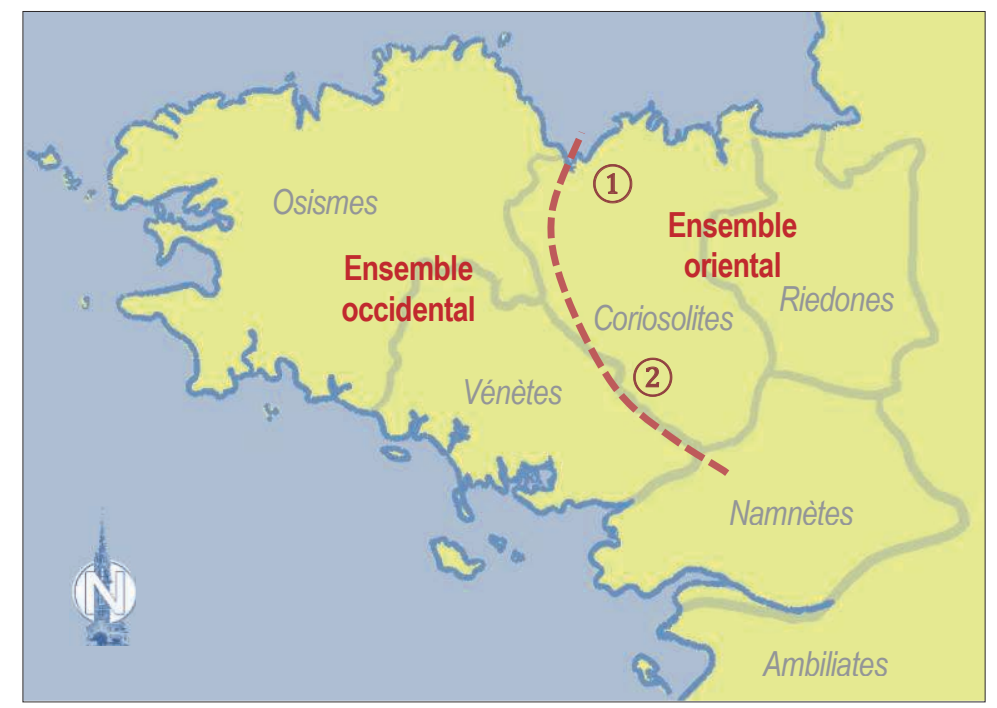

Carte 5 : Les espaces d'échanges culturels de fin de l'âge du fer et les limites de l'Armorique romaine.

Localisation des habitats du IIe_er siècle av. J.-C. situés le plus à l'ouest et présentant des mobiliers de type oriental : (1) la Tourelle, Lamballe (2) Bellevue, Augan

Riedones : population de civitates romaine.

En tiret, limite occidentale hypothétique de sites ayant livré des plaques de cuisson et des vases tronconiques à lèvre en « y ». En ligne grisée, limites des civitates de l'Armorique romaine.

\section{Convergence cartographique avec l'Armorique romaine}

Les civitates, villes et régions administrées par les Romains, s'appuieraient en principe sur des territoires nationaux. Il est donc pertinent de s'interroger sur la concordance géographique entre les civitates (carte 6) et la répartition actuelle des groupes de vannerie (carte 1). 


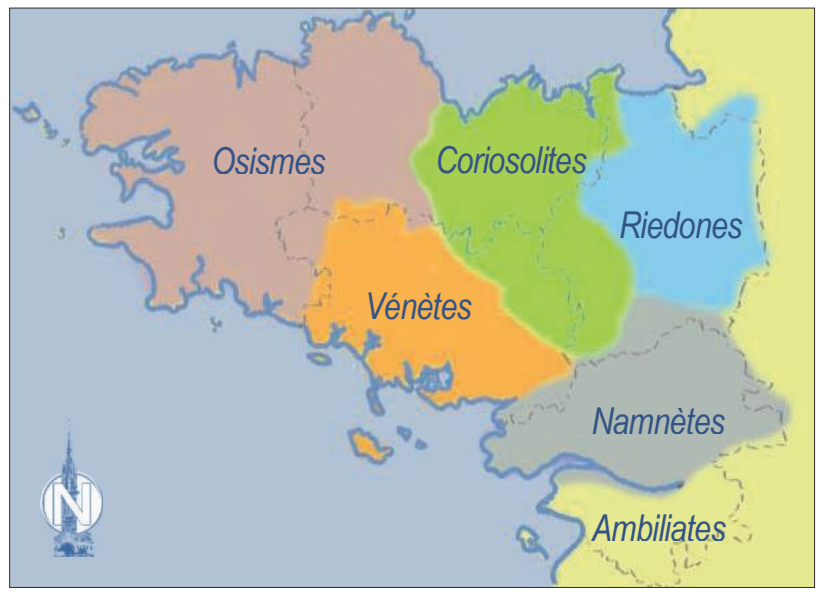

Carte 6 : Localisation des civitates de l'Armorique gallo-romaine.

En pointillé : limites actuelles des départements.

L'aire vénète correspond à celle où est aujourd'hui produite la vannerie du « groupe vannetais ».

L'aire des Osismes concorde avec les secteurs de production actuelle des variantes « bas cornouaillaise » et « nord Basse-Bretagne ».

La vannerie à montants croisés en lames de bois dite "groupehaut-breton » reprend quasi intégralement l'aire des Coriosolites et l'aire des Riedones.

La vannerie à montants courbés disposés en méridien s'accorde préférentiellement avec les territoires redones - où elle n'est dominante que sur sa marge - et namnètes.

Les cartes 6, 7 et 8 sont construites par superposition des limites des civitates avec les aires des noms issus de la valorisation de planches inédites de l'ALBRAM ${ }^{15}$. La carte 7 concerne la vannerie du groupe haut-breton.

15. Gabriel Guillaume, Jean-Paul Chauveau, Atlas Linguistique et Ethnographique, de Bretagne Romane, de l'Anjou et du Maine, Paris, CNRS, 1975-1983, 2 vol. parus. Les cartes numérotées 158, 159, 160 sont destinées au vol. 3, inédit. 


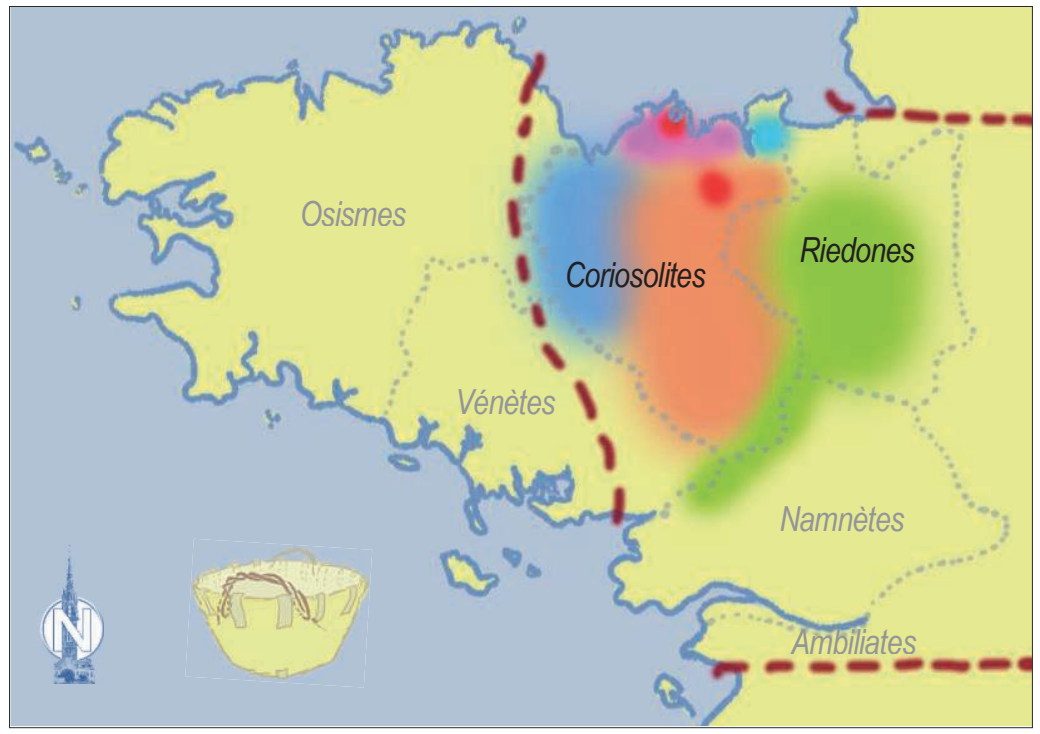

Carte 7 : Localisation des appellations des corbeilles à montants plats croisés disposés en hémiméridien.

Pointillés : limites des civitates de l'Armorique gallo-romaine

Tirets : limite de l'étude de l'étude ALBRAM

Nom vernaculaire d'une grande corbeille ronde selon ALBRAM3 :

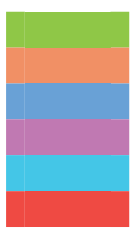

un carbasson, un calbasson, un cabasson
une cage, une caige, une caïge, un caigeo, un cageo, une cagette
une bine, une binelle, un bino, un bournô
une rondaïche, une rondaiche, une randajche
un potte
une canaïche, une canoche, le cano

Au sein de cet espace technique, la famille lexicale carbasson-calbassoncabasson forme une isoglosse qui se superpose avec les limites du secteur redones et le long de la Vilaine, voie de communication majeure. Le groupe géolinguistique bine-binelle-bino occupe le nord-ouest du secteur attribué aux Coriosolites, et le groupe lexical cage-caige-cache le sud et l'est. Le long de la Manche, d'autres formes lexicales sont utilisées dans des espaces très réduits : canaiche-canoche, potte ou rondaiche. Les corbeilles de ce groupe ne sont jamais mentionnées au-dehors des proches limites des civitates redones et coriosolites. Cette association entre groupe de vannerie, terminologie technique contemporaine et limites des civitates gallo-romain n'avait pas été jusqu’à présent décrite. 
La carte 8 présente la localisation des appellations des corbeilles à montants croisés disposés en méridien.

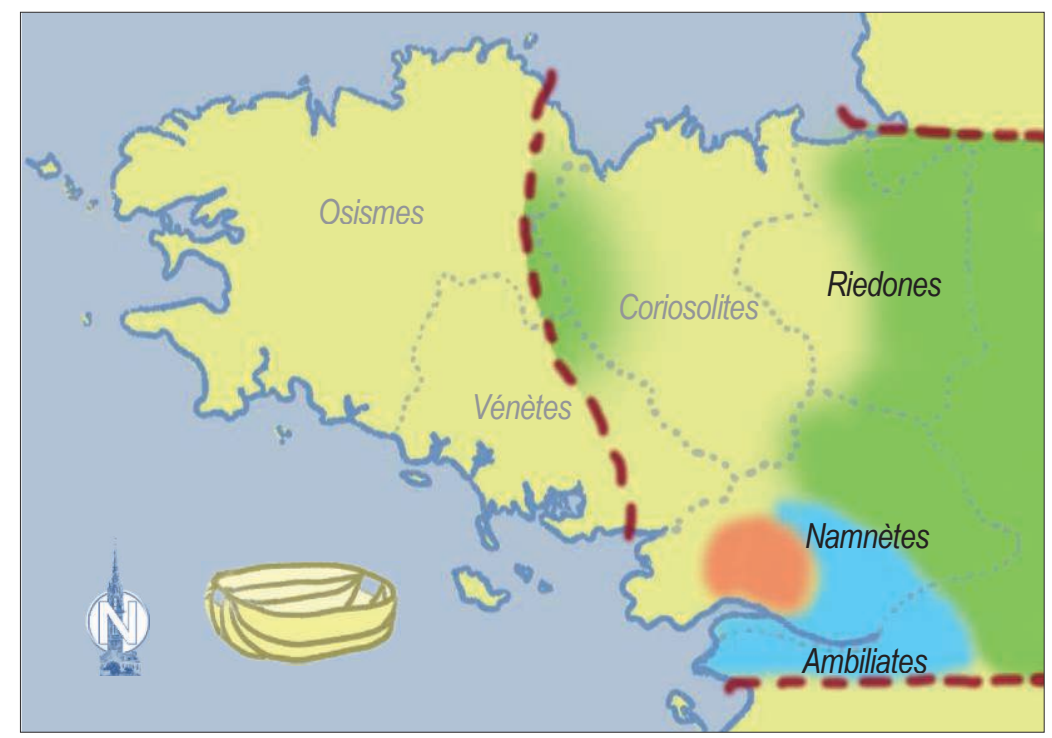

Carte 8 : Localisation des appellations des corbeilles à montants croisés disposés en méridien.

Pointillés : limites des civitates de l'Armorique gallo-romaine

Tirets : limite de l'étude de l'étude ALBRAM

Nom vernaculaire d'une grande corbeille ronde selon ALBRAM3 :

une resse, une reste, une rasse, une rèse, une résse, une reusse une hotte, des hottons

une balle, une banne, une benne

Cette diffusion par la périphérie s'oppose à celle des vanneries à montants disposés en hémiméridien, considérées comme natives. Les termes en resse-reste sont employés en Basse-Bretagne, mais l'usage a pu s'appliquer à des objets importés, les techniques locales ne permettant pas la production de ces vanneries de grand volume utilisées pour soigner les animaux.

La carte 9 présente les appellations des corbeilles utilisées pour faire lever la pâte à pain. 


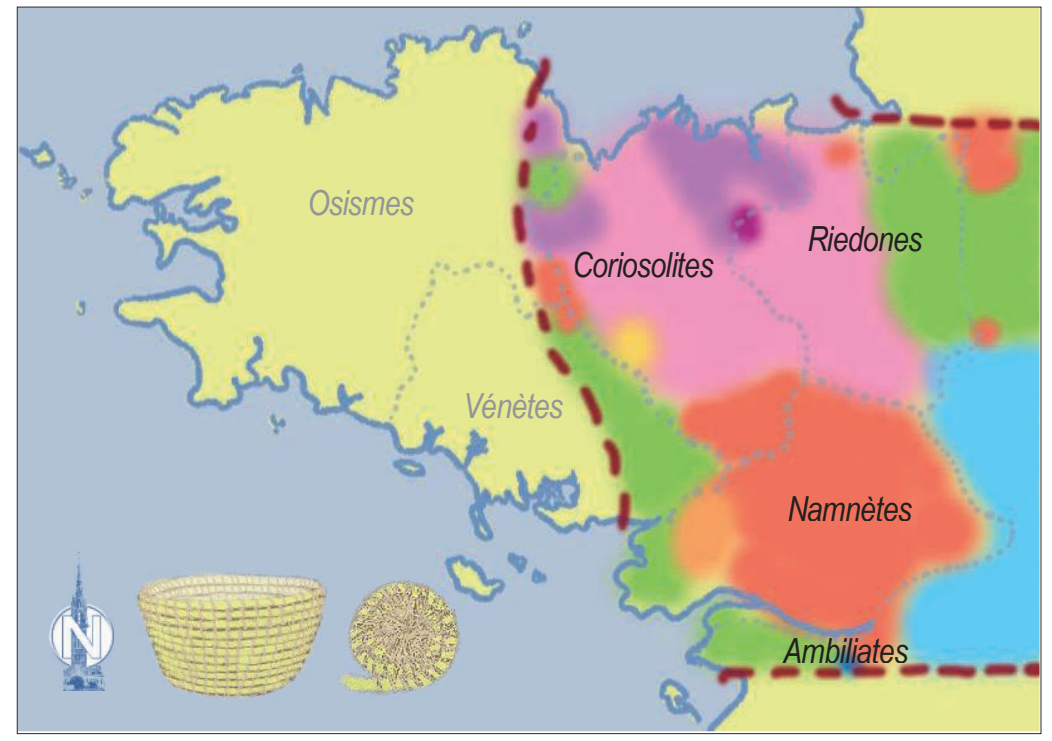

Carte 9 : Localisation des appellations des corbeilles à montant spiralé utilisées pour faire lever la pâte à pain.

Pointillés : limites des civitates de l'Armorique gallo-romaine,

Tirets : limite de l'étude de l'étude ALBRAM

Nom vernaculaire d'une grande corbeille ronde selon ALBRAM3 :

\begin{tabular}{|c|c|}
\hline & la binette, le binô, la binôche, la binelle, la bine à pain, le \\
\hline$O u^{*}$ & des panetons, des banetons, des binetons, des mannetons \\
\hline ou & un frontué, la frontouère \\
\hline ou & un puété, des puètuaw, un puatè, -tè \\
\hline & les paillons \\
\hline & la ruche, le ruchot, le runche ou runchot, bourriche ${ }^{* *}$, barruche ${ }^{* * *}$ \\
\hline & un joede, jède, jatte, jette, jəde, jède, un jədro, jadrau, des jados \\
\hline & un paillu, un pэillu, un pâillu \\
\hline & une guenotte \\
\hline
\end{tabular}

* sur le même secteur que isogloss bin-, sauf si cité dans le civitates Osismes

${ }^{* *}$ en sud-Loire ${ }^{* * *}$ exclusivement ouest du secteur Riedones

De l'examen de la carte, il ressort que les civitates redones et coriosolites s'associent bien aux termes lexicaux ayant un radical en bin-, et que dans le secteur des Namnètes ce sont les termes en jed- qui dominent à l'exception de la presqu'île guérandaise où ce mot revêt un autre sens. Le mot gède y désigne des récipients en bois tourné utilisés notamment pour la récolte du sel ${ }^{16}$. Dans ce secteur, on emploie les termes runche,

16. Pierre Lemonnier, Les salines de l'Ouest : logique technique, logique sociale, Paris, Éditions de la Maison des sciences de l'homme, 1980, p. 107. 
runchot pour désigner les corbeilles pour faire lever la pâte à pain. Ceci est à rapprocher de l'usage vannetais, aussi bien en secteur roman (carte 9) qu'en secteur celtique (carte 10, adaptation d'après le texte de Trépos $\left.{ }^{17}\right)$.

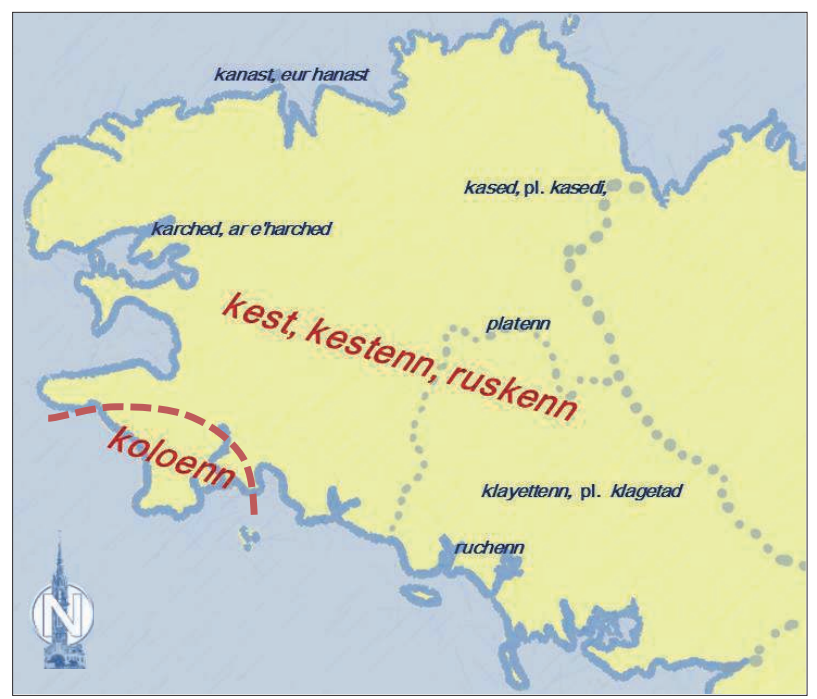

Carte 10 : Nom des corbeilles à montant spiralé utilisées pour faire lever la pâte à pain (d'après le texte de Trépos, 1926).

Pointillés : limites des civitates de l'Armorique gallo-romaine.

\section{Étymologie et aspects linguistiques}

Les correspondances entre territoires culturels anciens et des espaces de techniques et de terminologies vannières revêtent ici un intérêt particulier, car les termes employés sont possiblement des reliquats des langues autrefois parlées dans ces espaces. Ainsi le baskodenn, désignant un panier en Basse-Cornouaille (carte 11), est une appellation qui rappelle le terme gaulois bascauda, dont la signification aurait désigné un récipient tressé ${ }^{18}$. D'autres termes sont employés

17. Pierre TRÉPOS, Enquête sur le vocabulaire breton de la ferme, Rennes, Imprimeries réunies, $1962[1926]$.

18. Xavier Delamarre, Dictionnaire de la langue gauloise : Une approche linguistique du vieuxceltique continental, Paris, Errance, 2008 [2001], p. 68. 
en Basse-Bretagne pour désigner les corbeilles rondes : bouteg dans la partie nord, sklissen ou kolvenn dans le Vannetais, et en bas-vannetais kostinell ${ }^{19}$.

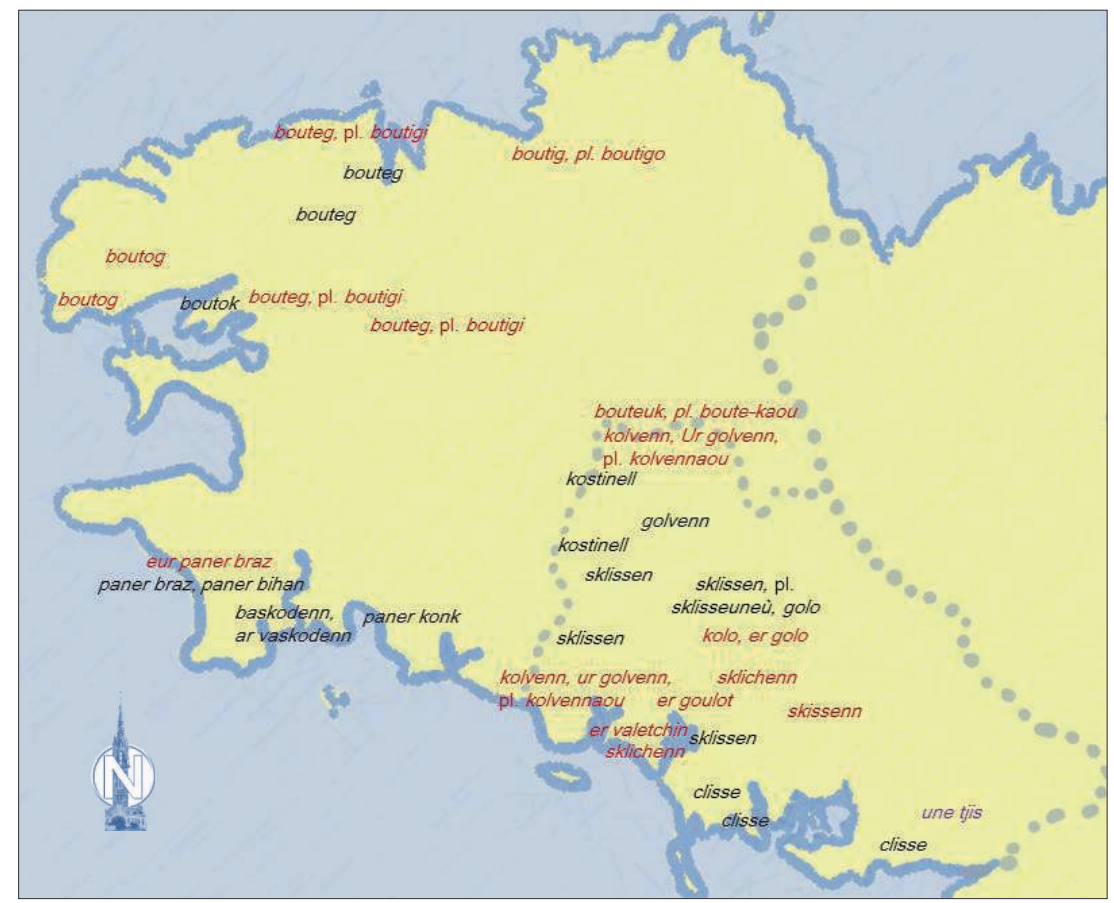

Carte 11 : Nom des vanneries natives de Basse-Bretagne.

Pointillés : limites des civitates de l'Armorique gallo-romaine.

Noms bretons : en noir Hérisset, 2012 ; en rouge Trépos, 1926 ; ALBRAM3 en violet.

Des travaux d'étymologie ont vu dans ces mots des emprunts à l'ancien français, du fait que le sens de la filiation est généralement établi entre les mots français et bretons selon l'antériorité de la citation, ici au bénéfice du français. Cependant les mots bretons cités n'ont pas la même signification que le terme français avec lequel il est mis en relation. Or, ils désignent localement sans équivoque un type de vannerie régional. Par exemple, des linguistes affilient bouteg à boute, outre, sklissen à esclice, éclat de bois et kostinell à coste, côte ${ }^{20}$. Cependant

19. Orthographié kostinel ou kochinel par Émile Ernault (1919).

20. Albert DeshaYes, Dictionnaire étymologique du breton, Douarnenez, Le Chasse-Marée, 2003, p. 129, 418, 665. 
ces rattachements hypothétiques nous aident à approcher l'origine véritable des termes étudiés, en envisageant une racine commune avec les mots français évoqués. Dans une langue ancienne, le nom de ces vanneries a pu être construit en considérant leur rôle de contenants, leur mode d'assemblage en côtes de bois, ou leur tressage fait d'éclisses. Le mot technique observé dans une langue locale a ainsi pu se conserver sans passage nécessaire par la langue française où il ne revêt pas le même sens.

L'ensemble de vanneries natives haut-breton (carte 7) se décompose en trois groupes géolinguistiques. Le groupe rennais carbasson (var. cabasson, calbasson) rappelle le gaulois carbenton, habituellement traduit par «char de guerre ». Xavier Delamarre ${ }^{21}$ note que des auteurs associent ce mot au français charpente (carpenta), désignant un «assemblage de pièces de bois », d'autres le rapprochent de la racine latine corbis « panier en osier ». Cette racine est par exemple présente dans le mot français corbeille (b. latin corbicula). Le mot provençal cabas désigne également un type de panier. Il est probable que cabas et carbasson, mots désignant un type précis d'objet tressé, ont à l'origine un sens générique se référant à un mode d'assemblage.

Le groupe géolinguistique briochin bine (var. bino, binelle, bourno) a peut-être une parenté avec le gaulois benna, traduit souvent par chariots et qui correspondrait à une voiture dont les caisses seraient tressées. Pour Jean-Paul Savignac ${ }^{22}$, benna s'associe à des objets tressés. Il cite banne, " corbeille ", et bagnole, de banniole, "la caisse du véhicule étant faite d'osier tressé comme une corbeille». Il rapproche ce mot du vieux gallois benn « chariot» et du vieil irlandais buinne « anneau, bracelet». Ces mots, selon Savignac, remontent «à une forme *bhendha, dérivée de la racine *bhendh- 'lier', cf. allemand binden 'lier', anglais bound 'lien'».

Le groupe géolinguistique cage (var. caige, caïge, cache, caiegeo, cago, cagette) est circonscrit sur un secteur se situant entre Dinan et Redon, et correspondant plus ou moins au territoire de l'ancien évêché de Saint-Malo. Cette forme lexicale peut se rapprocher du mot gaulois

21. X. Delamarre, op. cit., p. 105.

22. Jean-Paul SAVIGnAC, Dictionnaire Français-Gaulois, Paris, Snela-La différence, 2004, p. 56. 
cagio (haie, clôture), hypothèse acceptable si l'on se réfère à l'aspect clos de la corbeille. Selon Delamarre ${ }^{23}$, ce mot a de nombreux parents dans les langues celtiques : «le brittonique a le même mot : gallois cae 'haie, clôture', cornique ke 'haie', vieux breton ca 'baie, retranchement rempart', breton kae 'haie'. Forme de départ cagio d'une racine kagh- que l'on retrouve en latin caulae 'barrière d'une enceinte' ». Le mot français cage, désignant un contenant ajouré et fermé, est une autre illustration d'une évolution lexicale du sens générique de clôture, vers un sens particulier désignant un objet tressé précis.

Dans le secteur Coriosolites-Riedones, les corbeilles utilisées pour faire lever la pâte à pain sont désignées avec un vocabulaire ayant pour radical bin- (carte 8). Ces termes ont probablement la même origine que le mot bine commenté plus haut, même si ce mot renvoie dans le premier cas à la vannerie présentant des montants croisés en lames de bois. L'emploi de suffixes en -ette, -elle, -oche, -o exprime la dimension plus petite de la corbeille à pain : 30-35 cm d'ouverture contre environ $50 \mathrm{~cm}$ pour les corbeilles en bois. Elle est dite parfois bine à pain afin de bien la distinguer des bines ayant une autre destination. Il existe donc aussi un usage plus générique du mot bine, désignant les corbeilles rondes.

Pour dénommer le même type d'objet, les secteurs orientaux, voisins de la Normandie et du Maine, mais aussi le Vannetais et le littoral sud-breton emploient des variantes terminologiques de ruche (carte 8). Cette expression lexicale est souvent associée à une corbeille et rapprochée du mot latin riscia, riscus (panier), ou encore du grec rbiskos (coffre tressé), auquel on prête une origine celtique rusc- désignant l'écorce ou le panier ${ }^{24}$. Albert Deshayes ${ }^{25}$ associe les mots bretons, rusk, écorce et ruskenn, ruche ou panetière au vieux cornique rusc, au gallois rbisgl, à l'irlandais rusc, au gaëlique rùsg et au gaulois rusca. Les ruches destinées aux abeilles sont ordinairement réalisées en vannerie à montant spiralé, d'où peut-être ce nom lié à un basculement en français du générique vers le particulier.

23. X. Delamarre, op. cit., p. 96-97.

24. J.-P. SAVIGNAC, op. cit., p. 104.

25. A. Deshayes, op. cit., p. 637. 
Les panetières désignées par le groupe lexical jed- occupent l'aire des Namnètes (carte 9). Albert Deshayes ${ }^{26}$ rapproche le breton jedot (jale) du vieux français jadot (grande jatte). Une jale est un baquet ou une jatte, et les dictionnaires l'associent au gaulois galla (un contenant d'une certaine mesure). Émile Littré ${ }^{27}$ définit un jadot comme un « instrument en fer dont se servent les boulangers pour donner au pain la forme de couronne. " Il s'exprime ici un lien avec la confection du pain, tout pareillement aux panetières jed- du Nantais. Le mot français jatte, associé par les dictionnaires au latin găbăta (écuelle). La jatte est décrite habituellement comme un récipient de forme ronde très évasé, sans rebord ni anse. Cette définition peut inclure dans la forme les corbeilles pour faire lever la pâte à pain façonnées avec la technique de la vannerie spiralée. Les locuteurs du Pays nantais recourent à l'emploi exclusif des termes en jed-. Ce terme n'étant pas utilisé par les secteurs voisins, le groupe géolinguistique des mots en jed- participe donc à la définition d'un ensemble culturel nantais cohérent. Le secteur guérandais est exclu de cet ensemble, car il présente plutôt l'usage vannetais (runche) pour désigner les panetières.

Quant aux vanneries à montants disposés en méridien (carte 8), il est probable qu'un vocabulaire associé a accompagné la diffusion de ces techniques dans l'est de l'Armorique. Les termes recensés dans l'ALBRAM peuvent se diviser en quatre groupes.

Le groupe lexical resse (var. reste, rasse, rèse, résse, reusse) est peut-être une évolution différente de la forme celtique rusc- déjà citée, à l'instar de la forme latine riscia. Cette appellation est connue dans d'autres régions françaises (Flandre, Picardie, Normandie, Maine, Berry, Vendomois...) et en Belgique, et elle est souvent associée au vocabulaire technique de la mine et de la sidérurgie ${ }^{28}$.

Dans un secteur très limité de l'ouest du Nantais (carte 8), le mot hotte désigne un panier long. Une hotte est habituellement en français un panier pouvant être harnaché sur le dos. C'est un mot connu dans

26. Ibid., p. 350.

27. Émile LiTTRÉ, Dictionnaire de la Langue Française, Tome troisième I-Q, Paris, Hachette, 1883 , p. 166.

28. R. HÉRISSET, op. cit., p. 284-288. 
différentes langues germaniques et attesté en bas francique au XIII ${ }^{\mathrm{e}}$ siècle ${ }^{29}$.

Le mot balle désigne des paniers dans d'autres régions telles que le Lyonnais ${ }^{30}$. Dauzat ${ }^{31}$ propose un prototype celtique bala à relier à une racine indo-européenne $b h(e) l$-, gonfler, bouffer. Les objets en cuir que sont les balles découleraient de cette étymologie. D'autres contenants à la forme " gonflée » sont concernés par cette possible filiation comme les balles de foin, les ballots et probablement des corbeilles à la forme galbée.

Les mots banne et benne ont possiblement la même origine celtique que le terme bine analysé plus haut. D’une manière générale, le mot benne est un mot répandu pour désigner des récipients aussi bien tressés que façonnés dans le bois.

\section{Discussion : de l'origine de la singularité culturelle bretonne}

Les comparaisons réalisées avec des cartographies culturelles anciennes laissent entrevoir qu'en Haute et Basse-Bretagne la géographie de groupes de vanneries natifs se ferait donc dans la continuité d'espaces culturels antérieurs de plusieurs millénaires. L'hypothèse d'une persistance technique des vanneries natives s'accorde aussi avec celle d'une permanence dans le peuplement de la péninsule par les mêmes groupes de population. Cette approche s'accorde avec la théorie de la «Continuité Paléolithique ». Ainsi, concernant la présence de population celtique dans l'aire atlantique, Mario Alinei et Francesco Benozzo ${ }^{32}$ argumentent qu'elle serait constante depuis le Paléolithique supérieur, époque durant laquelle les îles britanniques et le Continent ne sont pas séparés par la mer.

29. Émile LiTTRÉ, Dictionnaire de la Langue Française, Tome deuxième D-H, Paris, Hachette, 1883, p. 2054.

30. Étienne MOLARD, Dictionnaire grammatical du manvais langage, ou recueil des expressions et des phrases vicieuses usitées en France, et notamment à Lyon, Lyon, s. n., 1803, p. 28.

31. Albert DauZat, Jean Dubois et Henri MitTERAND, Nouveau dictionnaire étymologique et bistorique, Larousse, Paris, 1968.

32. Mario Alinei et Francesco Benozo, «Les Celtes le long des côtes atlantique », dans D. Le Bris (dir.), Aires linguistiques, Aires culturelles : Études de concordances en Europe occidentale: zones Manche et Atlantique, Brest, CRBC, 2012, p. 55-76. 
Les singularités observées interrogent, dans la mesure où elles ne sont liées ni à la présence de reliefs marqués ni à une forte insularité. Il est légitime d'envisager que des phénomènes culturels singuliers, comme une vannerie particulière, soient la manifestation actuelle d'un isolement passé. L'hypothèse d'une géographie préhistorique plus clivante est à considérer. Les groupes de vannerie dominants en Europe sont singuliers au regard des continents voisins. Il est donc vraisemblable que ces techniques ne soient pas importées, mais indigènes. Elles se seraient alors développées à partir du peuplement du continent. De ce fait, l'origine et la diffusion de ces savoir-faire ont plausiblement eu lieu dans un contexte de période glaciaire. L'Europe s'étendait alors plus à l'Ouest, empiétant sur les limites actuelles de l'océan Atlantique. La Manche et la mer Celtique constituaient une vaste plaine qui reliait les Îles britanniques et l'Irlande au continent. De grands glaciers séparaient les groupes d'humains durant des périodes pouvant couvrir des centaines voire des milliers d'années. Certains groupes plus isolés ont développé des singularités culturelles fortes. Les vanniers préhistoriques armoricains ont dû mettre en œuvre une technique très singulière dans un isolat. Où était situé cet isolat? Sur le plateau continental de la mer Celtique ? Pour faire face à un climat très rigoureux, les populations préhistoriques se déplaçaient dans des espaces offrant suffisamment de ressources alimentaires. Une zone océanique présente à la fois l'accès à la nourriture et à un climat plus doux. En outre, une origine maritime des Armoricains conforterait l'hypothèse selon laquelle le casier à crustacés en forme de cloche soit un possible archétype d'une partie des vanneries natives bretonnes. L'impossible conservation des vanneries préhistoriques rend malheureusement improbable la découverte de vanneries artefacts, ce qui validerait les interprétations présentées.

\section{Perspectives et conclusion}

La géographie technique et lexicale de la vannerie armoricaine coïncide avec des zones d'échanges culturels anciennes, ce qui conforte l'hypothèse selon laquelle la répartition contemporaine des vanneries est un reliquat de cultures anciennes. La contribution de ce travail à la connaissance de la délimitation d'aires culturelles est dans le cas armo- 
ricain particulièrement féconde, du fait que les vanneries considérées présentent de fortes singularités techniques et sont plausiblement natives.

Au regard des techniques de vannerie, la Bretagne compte potentiellement trois sous-groupes appartenant à la vannerie à montants courbés disposés en hémiméridien. Elles occupent des espaces présentant des similitudes géographiques remarquables avec les civitates galloromaines peuplées par les Vénètes, les Osismes, les Coriosolites et les Riedones. La marche orientale de la péninsule se distingue par la présence ancienne d'un groupe technique différent dont les montants courbés sont disposés en méridien, partagé avec l'espace culturel Manche-Mer-duNord. Un autre groupe technique de vannerie apparenté, mais aux montures plus ramifiées, s'est répandu le long du littoral et vers l'intérieur de la péninsule à partir du sud et de l'est de la Bretagne, en suivant les fleuves.

En Bretagne, la persistance technique de la vannerie native a été favorisée par des conditions sociales ou géographiques propices au maintien des groupes de fabricants sur des aires de production stables. La persistance de techniques de vannerie se manifeste dans d'autres régions et l'étude des techniques peut devenir un matériau transactionnel entre ethnologues, linguistes et archéologues. Améliorer la connaissance géographique, technique et lexicale de la vannerie en Europe pourrait concourir à apporter des éléments complémentaires dans l'étude de la diffusion des techniques et la délimitation des espaces culturels.

Remerciements à Jean-Paul Chauveau (CNRS), à Stéphane Blanchet (Inrap) et à Jean-François Simon (UBO-CRBC). 


\section{Bibliographie}

Alinei Mario, BenOzZo Francesco «Les Celtes le long des côtes atlantiques : une présence ininterrompue depuis le Paléolithique ", dans D. Le Bris (dir.), Aires linguistiques, Aires culturelles : Études de concordances en Europe occidentale: zones Manche et Atlantique, Brest, CRBC, 2012, p. 55-76.

BALfET Hélène, "Vannerie ", Dictionnaire archéologique des techniques, Paris, Éditions de l'accueil, 1964, t. 2, p. 1033-1038.

Blanchet Stéphane (dir.), Lamballe, Côtes-d'Armor, ZAC de la Tourelle, Une occupation de longue durée sur le plateau de la Tourelle, Inrap, 2010, 650 p.

Blanchet Stéphane (dir.), Plouedern (Finistère) - Leslouc'h, Une longue occupation de la Protobistoire au Moyen-Âge, Inrap, 2013, 474 p.

Delamarre Xavier, Dictionnaire de la langue gauloise : Une approche linguistique du vieux-celtique continental, Paris, Errance, 2008 [2001], 440 p.

Deshayes Albert, Dictionnaire étymologique du breton, Douarnenez, Le ChasseMarée, 2003, 765 p.

Fiches Jean-Luc, StORDeur Danielle (dir.), coll., Tissage, Corderie, Vannerie, Centre de recherche d'archéologie du CNRS, Musée d'Archéologie d'Antibes, APDCA, Juan les pins, 1989, 218 p.

JAOUL Martine, GOLDSTEIN Bernadette, La Vannerie française, MNATP, Paris, RMN, 1990, 315 p.

Guillaume Gabriel, Chauveau Jean-Paul, Atlas Linguistique et Ethnographique, de Bretagne Romane, de l'Anjou et du Maine, Paris, CNRS, 1975-1983, 2 vol. parus. Les cartes numérotées 158, 159, 160 sont destinées au vol. 3, inédit.

HÉRISSET Roger, «Les vanniers en basse Cornouaille : des paniers pour les pêcheurs et les conserveries ", ArMen n ${ }^{\circ} 143$, Telgruc-sur-Mer, Fitamant, nov-déc. 2004, p. 40-45.

HÉRISSET Roger, Ethnologie des techniques de tressage en Bretagne. Matériaux pour une nouvelle approche classificatoire, thèse, CRBC-UBO, Brest, 2012, 816 p.

Hinguant S., Le Goff E., Jean S. et Marguerie D., « Le site gaulois de Bellevue à Augan (Morbihan). Un établissement rural en limite de deux influences armoricaines ", Revue archéologique de l'Ouest, nº 14, 1997, p. 5780.

HogAn Joe, Basketmaking in Ireland, Wickloow, Wordwell ltd, 2001, 300 p.

LALlier Dan, "Pierre Lelièvre, vannier à La-Chapelle-des-Marais", Monographies d'entreprises artisanales, G. H. Rivière et M. Maget (dir.), «Chantier $1810 », n^{\circ}$ 8, 1942, 116 p.

LALLIER Dan, «Les vanniers de Cancale, la vannerie pour la grande pêche », Monographies d'entreprises artisanales, G. H. Rivière et M. Maget (dir.), «Chantier $1810 », \mathrm{n}^{\circ}$ 58, 1952, $60 \mathrm{p}$. 
LE GOFF Elven, "Armorique" et "Bretagne" : réflexion sur l'assimilation de ces concepts pour la fin de l'âge du fer", Revue archéologique de l'Ouest, supp. $\mathrm{n}^{\circ} 10,2003$, p. 103-117.

LE RouZIC Suzanne, Matériaux ethnographiques et linguistiques pour la connaissance d'une population rurale de Basse-Bretagne : les riverains des forêts domaniales de Camors - Floranges - Lanvaux (Morbihan) et leur rapport à l'espace boisé, thèse, CRBC-UBO, 2007, 837 p.

LEMONNIER Pierre, Les salines de l'Ouest : logique technique, logique sociale, Éditions de la Maison des Sciences de l'Homme et de l'Université Lille III, 1980, $222 \mathrm{p}$.

Marcigny Cyril, Talon Marc «Sur les rives de la Manche. Qu'en est-il du passage de l'âge du Bronze à l'âge du Fer à partir des découvertes récentes ? ", De l'áge du Bronze à l'âge du Fer en France et en Europe occidentale $\left(X^{e}-V I I^{e}\right.$ av. J.-C.). La moyenne vallée du Rhône aux âges du Fer. Actes du $\mathrm{XXX}^{\mathrm{e}}$ colloque international de l'AFEAF, 2009, p. 385-403.

SAVIGnAC Jean-Paul, Dictionnaire Français-Gaulois, Paris, Snela-La différence, 2004, 336 p.

STORDEuR Danielle, "Vannerie et tissage au Proche-Orient néolithique: $\mathrm{XI}^{\mathrm{e}}-\mathrm{V}^{\mathrm{e}}$ millénaire ", Tissage, Corderie Vannerie - Actes des $\mathrm{XI}^{\mathrm{e}}$ rencontres internationales d'archéologie et d'histoire d'Antibes, APDCA, 1989, p. $19-40$.

TRÉPOS Pierre, Enquête sur le vocabulaire breton de la ferme, Rennes, Imprimeries Réunies, 1926.

WeNDRICH Willeke, The World According to Basketry, An Ethno-archaeological interpretation of basketry production in Egypt, CNWS Leiden Universiteit, 1999, $492 \mathrm{p}$. 